\title{
What do patients with psoriasis think about the causes of their disease and use for psoriasis?
}

\author{
Müzeyyen Gönül ${ }^{1, *}$, Seray Külcü Çakmak ${ }^{1}$, Ülker Gül ${ }^{1}$, Zeynep Bıyıklı \\ ${ }^{1}$ Ankara Numune Education and Research Hospital, 2nd Dermatology Clinic, Ankara, Turkey; ${ }^{2}$ Bsc, Ankara University Faculty of \\ Medicine, Biostatistic Department, Ankara, Turkey
}

\begin{abstract}
There are few reports about the beliefs of psoriasis patients related to their disorder and therapy modalities except for the medical ones. We aimed to investigate the beliefs of psoriasis patients about their disorders, where they had attended therapies unrelated to dermatologists and what modality of unconventional therapies they used for psoriasis in Turkey. The study was performed on 141 psoriasis patients. The age, sex, education status and the number of visits to the dermatology clinics were recorded. How the patients decided to visit the dermatology clinic (themselves, directed by others), where they attended for treatment of psoriasis outside of the dermatologist, what they think about the causes of their disease and what they used for treatment of psoriasis were asked. The mean age of the patients was 33.44 \pm 15.13 years. The patients whose ages were over 30 years significantly more went to dermatology clinics. $68.1 \%$ of the patients attended the dermatology clinic based on the individual decision, $18.4 \%$ were directed by family members and $5.7 \%$ by friends. $45.4 \%$ of the patients believed that stress was the cause of their disease. $54.6 \%$ of the patients had used at least one type of unconventional therapy for psoriasis. The most frequently used unconventional therapy forms were herbal $(24.1 \%)$ and spiritual therapies (22\%). One conclusion is psoriasis patients need education about their diseases. Dermatologists should have information about the tendencies of the patients and approach the patients with sensitivity and empathy and give objective knowledge about psoriasis.
\end{abstract}

Keywords psoriasis, unconventional therapy, beliefs, education

\section{INTRODUCTION}

Psoriasis is a common T-cell-mediated immune disorder. It occurs worlwide; however, the incidence varies from country to country (Fortune et al., 1998, Gudjonsson et al., 2008). While the exact cause of this process is unknown, certain environmental and genetic factors are known to be provoking factors. The classic presentation is well-defined red plaques with a silver scale. The characteristic scale makes the disorder highly visible and intrusive on the patient's lifestyle. The visible nature of the disease ensures that psoriasis has both physical and psychosocial effects (Fortune et al., 1998).

Topical corticosteroids, other immunosupressant drugs, oral retinoids, UV light, and several biological agents are used in the conventional approach to psoriasis. These treatments can be highly effective in the management of psoriasis, but none are universally safe and effective, and each has possible side effects (Traub and Marshall, 2007). Alternative natural therapies such as anti-inflammatory diet, elimination and/or rotation of allergenic foods, therapeutic fasting, nutritional supplementation, lifestyle interventions and the use of topical or systemic herbal formula provide some options with varying efficacy in the management of psoriasis (Traub and Marshall, 2007, Bartosińska et al., 2011, Treloar, 2010). Despite these treatment alternatives, psoriasis is still an incurable and chronic

*Correspondence: Müzeyyen Gönül

E-mail: muzeyyengonul@yahoo.com

Received June 15, 2011; Accepted February 13, 2012; Published

February 29, 2012

doi: http://dx.doi.org/10.5667/tang.2011.0024

C2012 by Association of Humanitas Medicine

TANG / www.e-tang.org disease (Bartosińska et al., 2011). As a result, the patients with psoriasis may pursue new approaches and develop a different idea about their disorders.

A few studies have been done about the beliefs of the patients with psoriasis related to their condition and what they used for the treatment of psoriasis (Fortune et al., 1998, Ryan 2008). We aimed to investigate the self beliefs of the psoriasis patients about their disorder, whom they attended besides dermatologists and what modality of therapy except the medical therapy they used for psoriasis in Turkey.

\section{MATERIALS AND METHODS}

The study included psoriatic patients successively attending the Ankara Numune Education and Research Hospital (Goverment Hospital), 2nd Dermatology Clinic in a six-month period. The hospital is in the city centre of the capital of Turkey, Middle Anatolia Region, and can be easily reached. It serves the patients of low-middle socioeconomomic levels attending from Middle and East Anatolia. The study was performed on patients with psoriasis whose ages were over 15 years because the information about the disease was taken from these patients. All of the patients' dermatologic examinations were done and their diagnoses were made clinically and histopathologically (if necessary). Also, the age, sex, education status, and number of applications to the dermatology clinics were recorded. The ages of the patients were grouped as $15-30,31-50$ and over 50 years. The education status was seperated into five groups: non-literates, graduation from primary school, secondary school, high school and university. The lengths of treatments

2012 / Volume 2 / Issue 1 / e8 


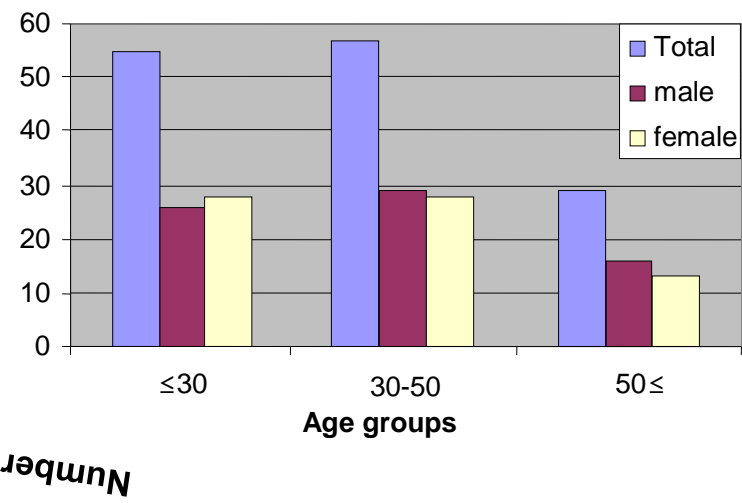

Fig.1. The three distribution of age groups according to sex.

were classified into two groups: under and over 10 years. How the patients decided to go to a dermatology clinic (themselves, directed by others), where they went for treatment of psoriasis besides dermatologists, what they think about the causes of their disease and what they used for the treatment of psoriasis were asked (Table 1). Statistical analysis of the data was done according to age, sex, and education status of the patients by chi-square tests. After the chi-square tests, a logistic regression analysis was done with parameters which had $p$ values lower than 0.20 .

\section{RESULTS}

One hundred and forty-one patients with psoriasis were enrolled in the study: $71(50.4 \%)$ were males and $70(49.4 \%)$ were females. The mean age of the patients were $33.44 \pm 15.13$ years (minimum 15, maximum 79 years). The distribution of the patients with psoriasis according to age groups and sex are seen in fig. 1. There was no statistical difference between the age groups according to sex.

The number of application of patients was divided into two groups as under $<10$ and $\geq 10$ and there were 70 (49.6\%) patients in the former group and $71(50.4 \%)$ patients in the latter group (Table 2). A statistical difference was not detected between both groups according to sex. When the number of applications was compared according to age groups, it was found that the patients whose ages were over 30 years significantly went more to the dermatology clinics $(p=0.01)$.

When the education statuses of the patients were evaluated, $7.8 \%$ of the patients were illiterate and the percentages of graduation from primary school, secondary school, high school

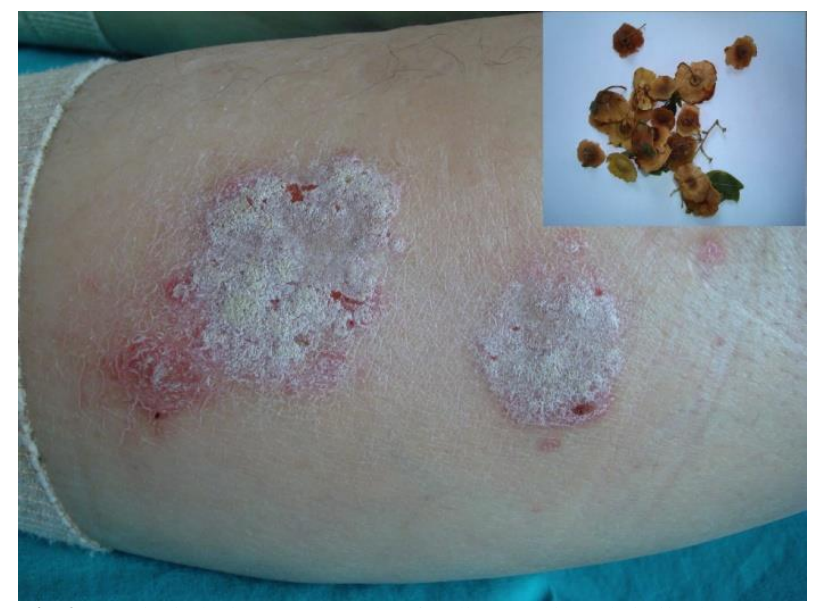

Fig.2. Psoriasis lesions and seeds of Paliurus spina-christii (the patient had drunk after. and university were $49.6 \%, 16.3 \%, 21.3 \%$ and $5 \%$ respectively. Education statuses of patients were divided into two groups for statistical analysis as the count of patients was small in each group: i) illiterate and graduated from primary school and ii) graduated from secondary school, high school and university. The first group consisted of $57.4 \%$ and second group consisted of $42.6 \%$ of the patients. Education statuses did not show any difference according to the sex of the patients.

$68.1 \%$ of the patients attended the dermatology clinic based on individual decision, $18.4 \%$ were advised by family members, $5.7 \%$ were advised by friends, $4.3 \%$ were directed by another doctor (nondermatologist), $2.1 \%$ by tv/radio and $0.7 \%$ by pharmacists. Sex, age groups and education statuses did not effect the modality of application to the dermatology clinic.

$45.4 \%$ of the patients had believed that stress was the cause of their disease. Beliefs of the patients about the reason of their disorders were as following: disorders of internal organs (7.8\%), disturbance of skin $(5.7 \%)$, microbial $(3.5 \%)$, nutritional (3.5\%), genetic (3\%), occupational (1.4\%), allergies and smoking ( $0.7 \%$ for each) (Table 3). Others $(29 \%)$ did not have any idea. There was no statistical relationship between the beliefs of patient and their sex, age groups and education status.

$47.5 \%$ of the patients applied elsewhere except the dermatology section for psoriasis. Of these patients, $22 \%$ had visited a spiritual healer, $21.3 \%$ had visited a herbalist, $12.8 \%$ a general practioner, $12.8 \%$ a pharmacist and $4.3 \%$ an internal specialist. The attendence choices, outside of the dermatologist, did not change according to sex, age groups and education statuses.

$54.6 \%$ of the patients had used at least one unconventional therapy for psoriasis. $63.4 \%$ of the patients who had used an unconventional therapy were males and $45.6 \%$ were females. Males had preferred unconventional therapy significantly more than females $(p=0.35)$ (Table 4). Although the use of unconventional therapy for psoriasis was higher in the $30-50$ year age group, this difference was not statistically significant. Also, the use of unconventional therapy did not change according to education statuses. Male patients used unconventional therapy 2.12 times more than female patients according to logistic regression analysis $[p<0.05, p=0.33$, odds ratio; 2.12, 95\% confidental interval (CI), 1.06 - 4.24]. The patients in the 31 - 50 age group used unconventional therapy more than other age groups $[p<0.05, p=0.038$, Odds ratio: $2.42,95 \% \mathrm{CI},(1.05$ - 5.60), logistic regression analysis]. Most frequently used unconventional therapy forms were herbal (24.1\%) (Fig. 2), spiritual (22\%), thermal spring (14.2\%), therapeutic regime $(8.5 \%)$, henna application $(8.5 \%)$, humectants $(5.7 \%)$, clay application $(2.1 \%)$, Kangal hot spring with fish $(2.1 \%)$ and soap (1.4\%). No significant difference was found between males and females in the use of unconventional therapy forms except that males visited the thermal springs more often than females $(p=0.017)$. Male patients and the patients in $31-50$ and $51<$ age groups significantly visited the thermal springs according to logistic regression analysis [respectively, $p<0.05, p=0.028$, odss ratio $3.46,95 \%$ CI, $(1.14-10.46), p<0.05, p=0.009$, Odds ratio: $6.77,95 \% \mathrm{CI},(1.62-28.23), p<0.05, p=0.041$, Odds ratio: $5.58,95 \%$ CI, (1.07 - 28.99)]. There was no significant difference between the use of unconventional therapy forms (except thermal spring) and age groups and education status.

\section{DISCUSSION}


Psoriasis affects both sexes equally. Although it may begin at any age, it is most likely to appear between the ages of 15 and 30 years (Gudjonsson, 2008). In our study, the male/female ratio was approximately $1(71 / 70)$. The mean age of the patients was $33.44 \pm 15.13$ years.

In our study, the number of visits to dermatology clinics for psoriasis were evaluated. Median visit count was 10 . The number of visits did not differ according to sex but the patients whose ages were over 30 years significantly attended more. This may be related to that the patients in this age group want to get over psoriasis as they want to marry and establish a business in these ages.

Of the patients, $68.1 \%$ went to the dermatology clinic based on an individual decision and $18.4 \%$ by the advice of family members. Sex, age groups and education statuses did not affect how the patients decided to attend the dermatology clinic. In the literature, we did not meet any information about this item. Our results showed that some psoriasis patients might ignore their conditions regardless of age, sex and education status until they are forced to so by others (friends/relatives) and/or they did not know which medical section they needed to attend for psoriasis in their country.

Fortune et al. found that the most commonly reported causative agents for psoriasis were stress $(60.1 \%)$ and genetic factors (55.5\%) (Fortune et al., 1998). Nevitt and Hutchinson reported that $40 \%$ of the patients with psoriasis were aware that psoriasis is a genetically determined disease and $37 \%$ thought that stress is an exacerbating factor (Nevitt and Hutchinson, 1996). In another study, it has been reported that $60 \%$ of the patients with psoriasis considered stress as the main cause for psoriasis exacerbation (Rigopoulus et al., 2010). In our study, $45.4 \%$ of the patients believed that stress was the cause of their disease. Beliefs of the patients about the reason of their disorders were as following: disorders of internal organs $(7.8 \%)$, disturbance of the skin $(5.7 \%)$, microbial $(3.5 \%)$, nutritional (3.5\%), genetic (3\%), occupational (1.4\%), allergic and smoking ( $0.7 \%$ for each). $29 \%$ of our patients did not have any idea about the causes of psoriasis. Even if the percent citing stress as the cause for psoriasis was not as high as in the results of Fortune et al. and Rigopoulus et al., it was in the first line (Fortune, 1998; Rigopoulus, 2010). The belief that genetic factors played a role in the pathogenesis was considerably lower than the literature in our study (Fortune, 1998).

$47.5 \%$ of the patients used treatments other than dermatology and these choices of psoriasis patients did not change according to sex, age group and education status. In our previous study which we investigated unconventional therapy use in dermatology outpatients, we detected that $14 \%$ of patients who used unconventional therapy were psoriasis patients and there was a statistically significant relationship between psoriasis and the use of unconventional therapy. Also, a significat relationship was detected between psoriasis and the use of herbal therapy, thermal springs, and spiritual healing (Gönül et al., 2009). Previously, the prevalence of unconventional therapy use in psoriasis had not been investigated in Turkey. In the present study, 54.6\% of the psoriasis patients had used at least one unconventional therapy for psoriasis. This rate is higher than the outcomes of Jensen $(42.5 \%)$ but lower than those of Ben-Arye (62\%) (Jensen, 1990; Ben-Arye, 2003). We found that males preferred unconventional therapy significantly more than females according to the literature (Jensen, 1990). The higher unconventional therapy use in males may be related to the more active social life of males because of their socioeconomic freedom in their country. Although the use of unconventional therapy for psoriasis was higher in the $30-50$ years group, this difference was not statistically significant. Unconventional therapy use did differ according to the education status.

Ben-Arye et al. reported that most often practiced modalities in psoriasis patients were herbal therapy, diet therapies, homeopathy and traditional Chinese medicine (Jensen, 1990). Jensen found that most used forms of unconventional therapy in psoriasis were health food preparations, herbal remedies, homeopathy and diet change (Jensen, 1990; Jensen, 1990). In our study, most frequently used unconventional therapy forms were herbal therapy, spiritual therapy, thermal springs, therapeutic regime and henna application. Homeopathy use was not seen in the study patients and the most commonly used herbal therapy was Paliurus spina-christii Mill. It is a perennial thorny shrub in Mediterranean region and Asia (Mosaddegh et al., 2004; Brantner et al., 1996) that has been used in folk medicine as a hypocholesterolemic agent. It has been shown that a Paliurus spina-christii extract can inhibit the rise of cholesterol and triglyceride in literature (Mosaddegh et al., 2004). Also, the remarkable antibacterial activity (mainly for Gram-positive bacteria) of its ethanolic extracts has been reported (Brantner et al., 1996). Cytokine secretion via bacterial stimulation is one of the theories responsible for the ethiopathogenesis of psoriasis (Traub and Marshall, 2007). Paliurus spina-christii can improve psoriasis lesions in some patients via its antimicrobial effect. Also, it can improve co-morbidities associated with psoriasis via the antilipidemic effects. Future studies may show some antiinflammatory activities that we currently do not know.

Spiritual therapy was used in $22 \%$ of our patients while it was not reported in the literature. We think that our patients had a desire for spiritual therapy because of their religion (Muslim). No significant difference was found in the use of unconventional therapy forms according to sex except the thermal springs. There was no significant difference in the use of unconventional therapy forms according to age groups and education status. However, males and the patients in the $31-50$ and $51<$ age groups significantly visited more often to the thermal springs than females and patients younger than 30 years. This discrepeancy may be due to that males and the patients in these age groups had more economic and social independence than females and younger patients.

We did not ask the patients why they used unconventional therapies and whether their dermatoses improved with unconventional therapies. We believe that the absence of satisfactory treatment for some dermatologic diseases such as psoriasis is an important reason for the patients to try unconventional therapies, as reported in the previous studies (Jensen, 1990; Magin et al., 2006).

Consequently, it is seen that psoriasis patients need education about their diseases in Turkey. We don't know how this condition is in other countries since similar studies have not yet been done. Our results present the first data about unconventional therapy of psoriasis in Turkey and show that males and patients older than 30 years use unconventional therapy more often. Our study shows that whatever the patients age and education status, the patients with psoriasis search new remedies for their health. Dermatologists should have information about the tendencies of the patients and approach the patients with sensitivity and empathy and give objective knowledge about the etiology, course and treatments of psoriasis.

\section{CONFLICT OF INTEREST}

The authors have no conflicting financial interests. 


\section{REFERENCES}

Bartosińska JP, Pietrzak A, Szepietowski J, Dreiher J, Maciejewski R, Chodorowska G. Traditional Chinese medicine herbs - are they safe for psoriatic patients? Folia Histochem Cytobiol. 2011;49:201-205.

Ben-Arye E, Ziv M, Frenkel M, Lavi I, Rosenman D. Complemantary medicine and psoriasis: linking the patient's Outlook with evidence-based medicine. Dermatology. 2003;207:302-307.

Brantner A, Males Z, Pepeljnjak S, Antolić A. Antimicrobial activity of Paliurus spina-christi Mill. (Christ's thorn). J Ethnopharmacol. 1996;52:119-122.

Fortune DG, Richards HL, Main CJ, Griffiths CE. What patients with psoriasis believe about their condition. J Am Acad Dermatol. 1998;39:196-201.

Gönül M, Gül U, Cakmak SK, Kiliç S. Unconventional medicine in dermatology outpatients in Turkey. Int J Dermatol. 2009;48:639-644.

Jensen P. Use of alternative medicine by patients with atopic dermatitis and psoriasis. Acta Derm Venereol. 1990;70:421424.

Jensen P. Alternative therapy for atopic dermatitis and psoriasis: patient-reported motivation, information source and effect. Acta Derm Venereol. 1990;70:425-428.

Magin PJ, Adams J, Heading GS, Pond DC, Smith W. Complemantary and alternative medicine therapies in acne, psoriasis, and atopic eczema: results of a qualitative study of patients' experiences and perceptions. J Altern Complement Med. 2006;12:451-457.

Mosaddegh M, Khoshnood MJ, Kamalinejad M, Alizadeh E. Study on the effect of Paliurus spina-christii on cholesterol, Trigliceride and HDL levels in diabetic male rats fed a high cholesterol diet. IJPR. 2004;3:51-54.

Nevitt GJ, Hutchinson PE. Psoriasis in the community: prevalence, severity and patients' beliefs and attitudes towards the disease. Br J Dermatol. 1996;135:533-537.

Rigopoulos D, Gregoriou S, Katrinaki A, Korfitis C, Larios G, Stamou C, Mourellou O, Petridis A, Rallis E, Sotiriadis D, Katsambas AD, Antoniou C. Characteristics of psoriasis in Greece: an epidemiological study of a population in a sunny Mediterranean climate. Eur J Dermatol. 2010;20:189-195.

Ryan S. Psoriasis: characteristics, psychosocial effects and treatment options. Br J Nurs. 2008;17:284-290.

Traub M, Marshall K. Psoriasis--pathophysiology, conventional, and alternative approaches to treatment. Altern Med Rev. 2007;12:319-330.

Treloar V. Integrative dermatology for psoriasis: facts and controversies. Clin Dermatol. 2010;28:93-99.

Wolf K, Goldsmith LA, Katz SI, Gilchrest BA, Paller AS, Leffell DJ. Fitzpatrick's Dermatology in General Medicine. $7^{\text {th }}$ ed. (New York, USA : Mc Graw Hill), pp. 169-193, 2008. 